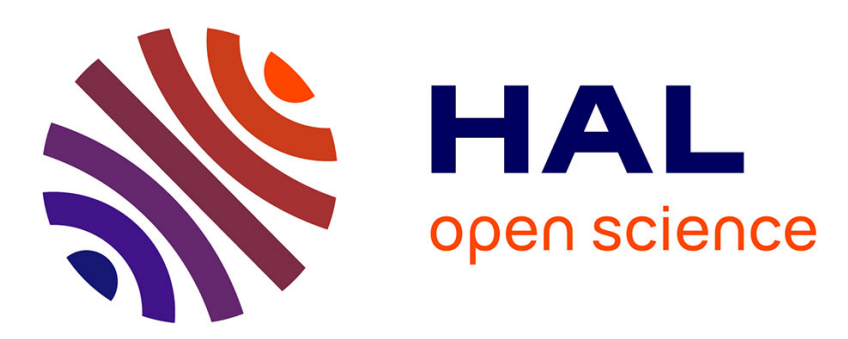

\title{
Observers design for one-sided Lipschitz discrete-time systems
}

Mohamed Benallouch, Mohamed Boutayeb, Michel Zasadzinski

\section{To cite this version:}

Mohamed Benallouch, Mohamed Boutayeb, Michel Zasadzinski. Observers design for onesided Lipschitz discrete-time systems. Systems and Control Letters, 2012, 61 (9), pp.879-886. 10.1016/j.sysconle.2012.05.005 . hal-00751553

\section{HAL Id: hal-00751553 https://hal.science/hal-00751553}

Submitted on 14 Nov 2012

HAL is a multi-disciplinary open access archive for the deposit and dissemination of scientific research documents, whether they are published or not. The documents may come from teaching and research institutions in France or abroad, or from public or private research centers.
L'archive ouverte pluridisciplinaire $\mathbf{H A L}$, est destinée au dépôt et à la diffusion de documents scientifiques de niveau recherche, publiés ou non, émanant des établissements d'enseignement et de recherche français ou étrangers, des laboratoires publics ou privés. 


\title{
Observers design for one-sided Lipschitz discrete-time systems
}

\author{
M. Benallouch, M. Boutayeb and M. Zasadzinski \\ Centre de Recherche en Automatique de Nancy (CRAN), Université de Lorraine, CNRS, \\ 186 rue de Lorraine, 54400 Longwy, FRANCE \\ E-mails : benallouch_mohamed@yahoo.fr, mohamed.boutayeb@univ-lorraine.fr , \\ michel.zasadzinski@univ-lorraine.fr
}

\begin{abstract}
This note focuses on state observer design for a general class of nonlinear discrete-time systems that satisfies the one-sided Lipschitz condition. It has been shown that this condition may encompass a large class of nonlinearities. However, challenging problems arise such as relevant choice of the Lyapunov function or non convexity of the obtained stability conditions. Both full-order and reducedorder observer design are considered. In this work, the main contribution is to provide first some mathematical artifacts on the Lyapunov function to obtain simple and workable stability conditions, furthermore we show how to obtain LMIs conditions to assure asymptotic convergence. On the other hand, we extend the obtained results to $n-p$ reduced order observer design. High performances are shown through simulation results.
\end{abstract}

Keywords : One-sided Lipschitz condition; Quadratic inner-boundedness; Nonlinear observers; Discretetime nonlinear systems; Linear matrix inequality (LMI) approach; Lyapunov stability.

\section{Introduction}

Over the past two decades, there has been significant research activity on observers design for nonlinear systems [1]-[2]-[3]-[4]-[5] and the references inside. This topic was motivated by the fact that state estimation can be used for control design, diagnosis or, more recently, synchronization and unknown input recovery [6]-[7]-[8]. It is worth to notice, however, that most of the existing results concern continuous time systems with few extensions to discrete-time ones [9]-[10]. As no universal approach exists, state observers, in particular for nonlinear systems, is still a challenging and open problem. Beside the famous extended Kalman filter, we distinguish a simple and useful nonlinear state observer that is based on the solution of a Riccati-like equation and the Lipschitz condition, we refer the reader to the pioneering works in [11]-[12] and their extensions [13]-[14]. In recent contributions [13]-[14], limitations of this approach have been highlighted. Indeed, it has been shown that the solution of the Riccati-like equation depends strongly on the Lipschitz constant, i.e. more the latter is larger, the more difficult it is to find a solution to the the Riccati-like equation.

In order to enlarge the domain of attraction and the class of nonlinear systems that can be considered, a useful and less general condition was recently introduced for observers design, that is the one sided Lipschitz condition. Interesting works on state observer design for this type of systems were recently developed in [15]-[16]-[17]-[18]-[19]-[20] however the asymptotic stability condition leads to a challenging problem that is the resolution of bilinear matrix inequalities. More recently, Abbaszadeh and Marquez. [20] have explored a more general Lipschitz condition with interesting mathematical artifacts to deduce LMI stability conditions. They show in particular inherent advantages with respect to the conservativeness induced by the classical Lipschitz condition. Inspired by their work we investigate here the problem of state observer design for one sided Lipschitz nonlinear discrete time systems. Indeed, it is worth to notice that the extension of the existing results on continuous time systems is a hard task and needs specific mathematical artifacts. 
First, we provide a general formulation of a quadratic Lyapunov function and construct an extended state vector to formulate the asymptotic stability condition in section 2 . On the other hand, we provide simple and useful mathematical manipulations to deduce sufficient conditions for asymptotic convergence in terms of linear matrix inequalities. Furthermore, we extend the obtained results to $(n-p)$ reduced order observer design in section 3. The latter may be interesting not only for real time applications but also may have less restrictive stability conditions. In the last section, relevant numerical examples are provided to show high performances of both techniques. Two illustrative examples are given in section 4 to show the efficiency of the proposed approach.

Notations. In a matrix, the notation $(\star)$ is used for the blocks induced by symmetry. $\langle x, y\rangle=x^{T} y$ is the scalar product. $\|x\|=\sqrt{\langle x, x\rangle}=\sqrt{x^{T} x}$ is the Euclidean vector norm. $|a|$ is the absolute value of the scalar $a . \lambda_{i}(A)$ is the $i^{\text {th }}$ eigenvalue of matrix $A$ and $\|A\|=\sqrt{\lambda_{\max }\left(A^{T} A\right)}$ is the induced 2-norm of matrix $A$. If $A=A^{T},\|A\|=\sqrt{\lambda_{\max }\left(A^{T} A\right)}=\left|\lambda_{\max }(A)\right|$. For a symmetric matrix $A, A>0$ means that the matrix $A$ is positive definite.

\section{Full-order observer design}

In this section, sufficient conditions for the existence of an observer are derived and a design procedure is presented. Let us consider the nonlinear system

$$
\left\{\begin{aligned}
x(k+1) & =A x(k)+f(x(k), y(k)) \\
y(k) & =C x(k)
\end{aligned}\right.
$$

where $x(k) \in \mathbb{R}^{n}$ and $y(k) \in \mathbb{R}^{p}$ denotes respectively the state and the linear output. $A$ and $C$ are constant matrices of adequate dimensions. $f: \mathbb{R}^{n} \times \mathbb{R}^{p} \rightarrow \mathbb{R}^{n}$ is a real nonlinear vector field.

Our objective is to design an asymptotic observer from the measured output signals $y(k)$ to estimate the state $x(k)$. The following assumptions are made throughout this paper.

\section{Assumption 1.}

1. $f$ is one-sided Lipschitz with respect to $x(k)$. i.e,

$$
\langle f(x, y)-f(\hat{x}, y), x-\hat{x}\rangle \leqslant \rho\|x-\hat{x}\|^{2}, \quad \text { for any } x, \hat{x} \in \mathbb{R}^{n}, y \in \mathbb{R}^{p}
$$

where $\rho$ is the so-called one-sided Lipschitz constant which can be positive or negative.

2. $f$ is quadratically inner-bounded with respect to $x(k)$. i.e,

$$
\|f(x, y)-f(\hat{x}, y)\|^{2} \leqslant \beta\|x-\hat{x}\|^{2}+\gamma\langle x-\hat{x}, f(x, y)-f(\hat{x}, y)\rangle .
$$

where $\beta$ and $\gamma$ are real scalars.

Unlike the well-known Lipschitz condition, the constants $\rho, \beta$ and $\gamma$ can be positive, negative or zero. In addition, if the function $f$ is Lipschitz, then it is also both one-sided Lipschitz and quadratically inner-bounded $(\beta>0$ and $\gamma$ ), but the converse is not true (see [20]). The one-sided Lipschitz condition $(2)$, considered in [15] and [18], provides a less conservative condition than the classical Lipschitz one. The concept of quadratic inner-boundedness (3), given in [20], is very useful to provide tractable LMI stability conditions.

The observer of system (1) is defined by the following form

$$
\hat{x}(k+1)=A \hat{x}(k)+f(\hat{x}(k), y(k))+K(y(k)-C \hat{x}(k))
$$

where $\hat{x}(k)$ denotes the estimate of the state vector $x(k)$ and $K$ is the gain matrix to be computed. Let $e(k)=x(k)-\hat{x}(k)$. Then from the observer (4) and the system (1), the dynamics of the state estimation error is described by

$$
e(k+1)=(A-K C) e(k)+\Delta f_{k}
$$

where $\Delta f_{k}=f(x(k), y(k))-f(\hat{x}(k), y(k))$.

Using the above assumption, the following theorem provides sufficient conditions so that equation (4) is an asymptotic full-order observer for system (1). 
Theorem 1. Under assumption 1, the system (4) is an asymptotic observer for system (1) if there exist scalars $\alpha>0, \mu_{1}>0, \mu_{2}>0, \rho, \beta, \gamma$ and $\epsilon>0$ and matrices $P=P^{T}>\alpha I_{n}, Q=Q^{T}>0, S$ and $X$ that solve the following $L M I$

$$
\left[\begin{array}{ll}
P & S \\
S^{T} & Q
\end{array}\right]>0
$$

and

$$
\mathbb{N}<0
$$

where $\mathbb{N}$ is given by

$$
\mathbb{N}=\left[\begin{array}{cccccc}
\mathbb{N}_{11} & \mathbb{N}_{12} & 0 & \mathbb{N}_{14} & \mathbb{N}_{14} & 0 \\
\star & \mathbb{N}_{22} & \mathbb{N}_{23} & 0 & 0 & 0 \\
\star & \star & \mathbb{N}_{33} & 0 & 0 & \mathbb{N}_{23}^{T} \\
\star & \star & \star & -\eta^{-1} P & 0 & 0 \\
\star & \star & \star & \star & -\epsilon I_{n} & 0 \\
\star & \star & \star & \star & \star & -\epsilon^{-1} \alpha^{2} I_{n}
\end{array}\right]
$$

with

$$
\left\{\begin{array}{l}
\eta=1+2(|\beta|+|\rho|) \\
\mathbb{S}=S-\left(\mu_{1} \gamma-\mu_{2}\right) I_{n} \\
\mathbb{N}_{11}=-P+2\left(\mu_{1} \beta+\mu_{2} \rho\right) I_{n} \\
\mathbb{N}_{12}=\eta A^{T} P-\eta C^{T} X-\mathbb{S} \\
\mathbb{N}_{14}=A^{T} P-C^{T} X \\
\mathbb{N}_{22}=\eta P-Q-2 \mu_{1} I_{n} \\
\mathbb{N}_{23}=S+\alpha(\gamma-1) I_{n} \\
\mathbb{N}_{33}=Q-2 \alpha I_{n}
\end{array}\right.
$$

Then, the gain for observer is given by $K=P^{-1} X^{T}$.

Proof. Let us consider the quadratic Lyapunov function

$$
V_{k}=\left[\begin{array}{c}
e(k) \\
\Delta f_{k}
\end{array}\right]^{T}\left[\begin{array}{cc}
P & S \\
S^{T} & Q
\end{array}\right]\left[\begin{array}{c}
e(k) \\
\Delta f_{k}
\end{array}\right]
$$

where $\Delta f_{k}$ defined in (5) and $\left[\begin{array}{cc}P & S \\ S^{T} & Q\end{array}\right]>0$. Moreover, the variation $\Delta V=V_{k+1}-V_{k}$ of this Lyapunov function is given by

$$
\Delta V=e^{T}(k+1) P e(k+1)-e^{T}(k) P e(k)-\Delta f_{k}^{T} Q \Delta f_{k}+\Delta f_{k+1}^{T} Q \Delta f_{k+1}+2 e^{T}(k+1) S \Delta f_{k+1}-2 e^{T}(k) S \Delta f_{k} .
$$

The one-sided Lipschitz and the quadratically inner-bounded conditions (2) and (3) give the following inequality

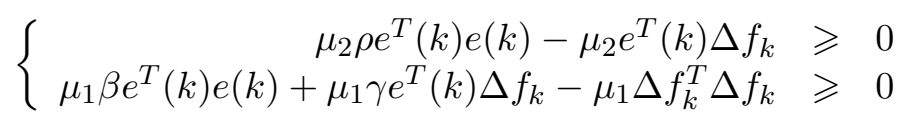

where $\mu_{1}$ and $\mu_{2}$ are arbitrary strictly positive scalars.

The following inequality is obtained by adding the left hand side of (12) to (11)

$$
\begin{aligned}
& \Delta V \leqslant e^{T}(k+1) P e(k+1)+e^{T}(k)\left(-P+2\left(\mu_{2} \rho+\mu_{1} \beta\right) I\right) e(k) \\
& \quad+2 e^{T}(k+1) S \Delta f_{k+1}-2 e^{T}(k)\left(S+\left(\mu_{2}-\mu_{1} \gamma\right) I\right) \Delta f_{k}-\Delta f_{k}^{T}\left(Q+2 \mu_{1} I\right) \Delta f_{k}+\Delta f_{k+1}^{T} Q \Delta f_{k+1} .
\end{aligned}
$$

On the other hand, using the one-sided Lipschitz and the inner-bounded conditions (2) and (3) with the fact that $P>\alpha I_{n}$, it follows that

$$
\begin{aligned}
& |\rho| e^{T}(k+1) P e(k+1)-\alpha e^{T}(k+1) \Delta f_{k+1}>\alpha|\rho| e^{T}(k+1) e(k+1)-\alpha e^{T}(k+1) \Delta f_{k+1} \geqslant 0 \\
& |\beta| e^{T}(k+1) P e(k+1)+\alpha \gamma e^{T}(k+1) \Delta f_{k+1}-\alpha \Delta f_{k+1}^{T} \Delta f_{k+1}
\end{aligned}
$$




$$
>\alpha|\beta| e^{T}(k+1) e(k+1)+\alpha \gamma e^{T}(k+1) \Delta f_{k+1}-\alpha \Delta f_{k+1}^{T} \Delta f_{k+1} \geqslant 0 .
$$

Thus, by adding the left terms in inequalities (14) and (15) to (13), we get

$$
\begin{aligned}
\Delta V \leqslant \eta e^{T}(k+1) P e(k+1)+e^{T}(k)( & \left.P+2\left(\mu_{2} \rho+\mu_{1} \beta\right) I\right) e(k)-\Delta f_{k}^{T}\left(Q+2 \mu_{1} I\right) \Delta f_{k}-2 e^{T}(k)\left(S+\left(\mu_{2}-\mu_{1} \gamma\right) I\right) \Delta f_{k} \\
& +2 e^{T}(k+1)(S+\alpha(\gamma-1) I) \Delta f_{k+1}+\Delta f_{k+1}^{T}(Q-2 \alpha I) \Delta f_{k+1}
\end{aligned}
$$

Using the dynamics of the estimation error (5) and based on the Lyapunov stability theory, the convergence of the estimation error is guaranteed, as soon as $\Delta V<0$ is negative definite, which holds if

$$
\xi^{T} \mathcal{N} \xi<0
$$

where

$$
\xi^{T}(k)=\left[\begin{array}{lll}
e^{T}(k) & \Delta f_{K}^{T} & \Delta f_{K+1}^{T}
\end{array}\right]
$$

and

$$
\mathcal{N}=\left[\begin{array}{ccc}
\mathbb{N}_{11}+\eta \mathcal{N}_{12} P^{-1} \mathcal{N}_{12}^{T} & \eta \mathcal{N}_{12}-\mathbb{S} & \mathcal{N}_{12} P^{-1} \mathbb{N}_{23} \\
\star & \mathbb{N}_{22} & \mathbb{N}_{23} \\
\star & \star & \mathbb{N}_{33}
\end{array}\right]
$$

with $\mathcal{N}_{12}=(A-K C)^{T} P$.

The BMI problem of (17)-(19) is not convex and the existing LMI computational procedures can not be applied. To linearize the BMI problem (17)-(19), we proceed in three steps.

The first step concerns matrix $\mathcal{N}_{12} P^{-1} \mathbb{N}_{23}$. Since we have $\mathcal{N}_{12}$ and $\mathbb{N}_{23}$ acting separately in (19), and $\mathcal{N}_{12}$ is a function of $K$ and $P$, it is necessary to rewrite $\mathcal{N}_{12} P^{-1} \mathbb{N}_{23}$ under a form so that $\mathcal{N}_{12}$ and $\mathbb{N}_{23}$ appear separately in this equation. Notice that the matrix $\mathcal{N}$ can be rewritten as follows

$$
\mathcal{N}=\overline{\mathcal{N}}+\Psi \Phi^{T}+\Phi \Psi^{T}
$$

where

$$
\overline{\mathcal{N}}=\left[\begin{array}{ccc}
\mathbb{N}_{11}+\eta \mathcal{N}_{12} P^{-1} \mathcal{N}_{12}^{T} & \eta \mathcal{N}_{12}-\mathbb{S} & 0 \\
\star & \mathbb{N}_{22} & \mathbb{N}_{23} \\
\star & \star & \mathbb{N}_{33}
\end{array}\right]
$$

and

$$
\begin{aligned}
\Psi^{T} & =\left[\begin{array}{lll}
\mathcal{N}_{12}^{T} & 0 & 0
\end{array}\right] \\
\Phi^{T} & =\left[\begin{array}{lll}
0 & 0 & P^{-1} \mathbb{N}_{23}
\end{array}\right] .
\end{aligned}
$$

In the second step, we use the well-known matrix inequality

$$
\Psi \Phi^{T}+\Phi \Psi^{T} \leqslant \epsilon \Phi \Phi^{T}+\frac{1}{\epsilon} \Psi \Psi^{T}
$$

with $\epsilon>0[21]$ to obtain an upper bound of the term $\Psi \Phi^{T}+\Phi \Psi^{T}$ in (20) and the following inequality holds for any scalar $\epsilon>0$

$$
\Psi \Phi^{T}+\Phi \Psi^{T} \leqslant \epsilon\left[\begin{array}{c}
0 \\
0 \\
\mathbb{N}_{23}^{T} P^{-1}
\end{array}\right]\left[\begin{array}{lll}
0 & 0 & P^{-1} \mathbb{N}_{23}
\end{array}\right]+\frac{1}{\epsilon}\left[\begin{array}{c}
\mathcal{N}_{12} \\
0 \\
0
\end{array}\right]\left[\begin{array}{lll}
\mathcal{N}_{12}^{T} & 0 & 0
\end{array}\right] .
$$

From the fact that $P^{-2}<\alpha^{-2} I$ (since $P>\alpha I$ ), it follows that

$$
\mathcal{N} \leqslant \overline{\mathcal{N}}+\epsilon \alpha^{-2}\left[\begin{array}{c}
0 \\
0 \\
\mathbb{N}_{23}^{T}
\end{array}\right]\left[\begin{array}{lll}
0 & 0 & \mathbb{N}_{23}
\end{array}\right]+\frac{1}{\epsilon}\left[\begin{array}{c}
\mathcal{N}_{12} \\
0 \\
0
\end{array}\right]\left[\begin{array}{lll}
\mathcal{N}_{12}^{T} & 0 & 0
\end{array}\right]=\mathbf{N}
$$


This implies that if the linear matrix inequality

$$
\mathbf{N}=\left[\begin{array}{ccc}
\mathbb{N}_{11}+\Upsilon^{T} \Pi \Upsilon & \eta \mathcal{N}_{12}-\mathbb{S} & 0 \\
\star & \mathbb{N}_{22} & \mathbb{N}_{23} \\
\star & \star & \mathbb{N}_{33}+\epsilon \alpha^{-2} \mathbb{N}_{23}^{T} \mathbb{N}_{23}
\end{array}\right]<0
$$

is verified then $\Delta V<0$, where

$$
\Upsilon^{T}=\left[\begin{array}{ll}
\mathcal{N}_{12} & \mathcal{N}_{12}
\end{array}\right] \text { and } \Pi=\left[\begin{array}{cc}
\eta P^{-1} & 0 \\
0 & \frac{1}{\epsilon} I
\end{array}\right]
$$

Now using the Schur lemma [22], the inequality (24) is equivalent to the following LMI

$$
\left[\begin{array}{ccccc}
\mathbb{N}_{11} & \eta \mathcal{N}_{12}-\mathbb{S} & 0 & \Upsilon^{T} & 0 \\
\star & \mathbb{N}_{22} & \mathbb{N}_{23} & 0 & 0 \\
\star & \star & \mathbb{N}_{33} & 0 & \mathbb{N}_{23}^{T} \\
\star & \star & \star & -\Pi^{-1} & 0 \\
\star & \star & \star & \star & -\epsilon^{-1} \alpha^{2} I_{n}
\end{array}\right]<0 .
$$

By using the expression $\Upsilon$ and $\Pi$ given by (25) and the notation $X=K^{T} P$, the LMIs in (7) and (26) are equivalent, which completes the proof.

Remark 1. As pointed in [20] in the continuous-time case, the detectability of the pair $(A, C)$ is not required due to the use of assumption 1. Then, the "linear part" of the observation error must not necessarily dominate the "nonlinear" one. A consequence of this is that we get less conservative results than those derived with a Lipschitz condition.

\section{Reduced-order observer design}

In this section we present a method to design a reduced-order observer for system (1), i.e. an observer which does not estimate the full state $x(k)$, but only a linear combination of $x(k)$ given by the reduced state vector $z(k) \in \mathbb{R}^{n-p}$ defined as [23]

$$
z(k)=L x(k)
$$

where $L \in \mathbb{R}^{(n-p) \times n}$ is a full row rank matrix. Without loss of generality, matrix $C$ is of full row rank,

then the matrix $L$ can always be chosen such that $\left[\begin{array}{l}L \\ C\end{array}\right]$ is nonsingular, so matrices $M$ and $N$ can be defined as $\left[\begin{array}{l}L \\ C\end{array}\right]^{-1}=\left[\begin{array}{ll}N & M\end{array}\right]$. We then have

$$
x(k)=N z(k)+M y(k) .
$$

From (1), (27), and (28), we obtain the following nonlinear reduced form

$$
z(k+1)=A_{z} z(k)+\operatorname{Lg}(z(k), y(k))+B_{z} y(k)
$$

where $A_{z}=L A N, B_{z}=L A M$ and $g(z(k), y(k))=f(N z(k)+M y(k), y(k))$.

Since the nonlinear map $f(x(k), y(k))$ is one-sided Lipschitz and quadratically inner-bounded, then also the function $g(z(k), y(k))$ is one-sided with constants $\rho_{g}$ and quadratically inner-bounded, with constants $\beta_{g}$ and $\gamma_{g}$.

We propose a reduced-order observer corresponding to (29) as follows

$$
\left\{\begin{aligned}
\hat{z}(k+1) & =A_{z} \hat{z}(k)+L g(\hat{z}(k), y(k))+B_{z} y(k)+K\left(y(k+1)-C \hat{x}_{x+1 / k}\right) \\
\hat{x}_{x+1 / k} & =A N \hat{z}(k)+A M y(k)+g(\hat{z}(k), y(k)) .
\end{aligned}\right.
$$

By setting $C_{z}=C A N$, we obtain

$$
y(k+1)=C_{z} z(k)+C A M y(k)+C g(z(k), y(k))
$$


and we deduce

$$
\varepsilon(k+1)=\left(A_{z}-K C_{z}\right) \varepsilon(k)+(L-K C) \Delta g_{k}
$$

where $\varepsilon(k)=z(k)-\hat{z}(k)$ and $\Delta g_{k}=g(z(k), y(k))-g(\hat{z}(k), y(k))$.

Using the assumption 1 , an asymptotic reduced-order observer for system (1) is given by the system (30) if the conditions given in the following theorem hold.

Theorem 2. Under assumption 1, system (30) is an observer for system (29), if there exist scalars $\alpha>0, \mu_{1}>0, \mu_{2}>0, \rho, \beta, \gamma$ and $\epsilon>0$ and matrices $P=P^{T}>\alpha I_{n}, Q=Q^{T}>0, S$ and $X$ that solve the following convex optimization problem:

$$
\left[\begin{array}{ll}
P & S \\
S^{T} & Q
\end{array}\right]>0
$$

and

$$
\mathbb{M}<0
$$

where $\mathbb{M}$ is given by (36), with

$$
\left\{\begin{array}{l}
\eta=1+2(|\beta|+|\rho|) \\
\mathbb{M}_{23}=S+\alpha(\gamma-1) I_{n} \\
\mathbb{S}=-N^{T} S+\left(\mu_{1} \gamma-\mu_{2}\right) N^{T} \\
\mathbb{M}_{11}=-N^{T} P N+2\left(\mu_{1} \beta+\mu_{2} \rho\right) N^{T} N \\
\mathbb{M}_{22}=-\left(Q+2 \mu_{1} I_{n}\right) \\
\mathbb{M}_{33}=Q-2 \alpha I_{n}
\end{array}\right.
$$

Then, the gain for observer is given by $K=L P^{-1} X^{T}$.

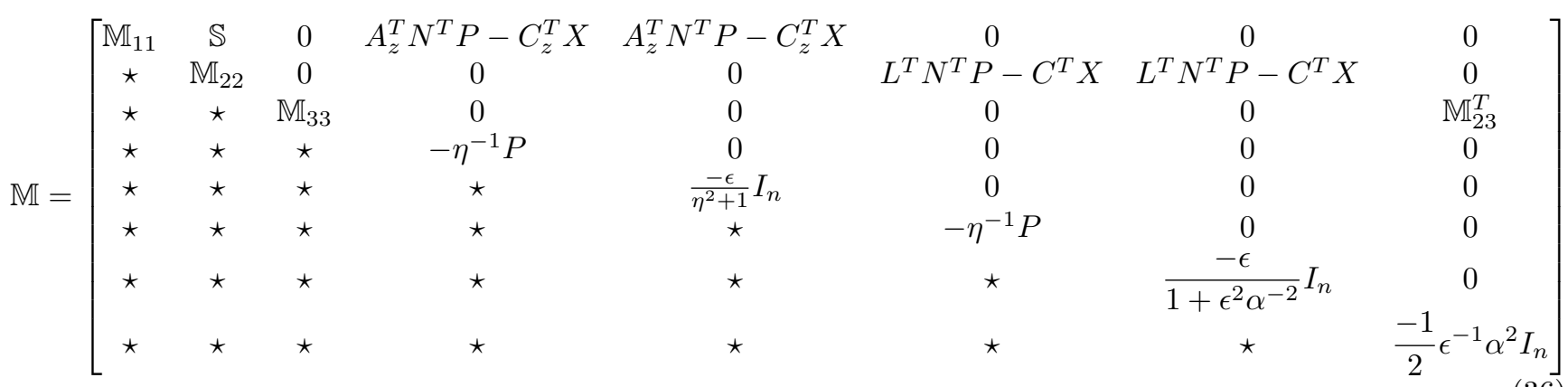

Proof. The proof of this theorem can be achieved by the use of the following Lyapunov function

$$
V_{k}=\left[\varepsilon(k)^{T} \quad \Delta g_{k}^{T}\right]\left[\begin{array}{cc}
N^{T} P N & N^{T} S \\
S^{T} N & Q
\end{array}\right]\left[\begin{array}{c}
\varepsilon(k) \\
\Delta g_{k}
\end{array}\right]
$$

where $\left[\begin{array}{cc}P & S \\ S^{T} & Q\end{array}\right]>0$

Following similar steps (11)-(16) as in the previous proof and by replacing $e(k)$ and $f_{k}$ by $N \varepsilon(k)$ and $g_{k}$, respectively, the convergence of the estimation error is guaranteed, if $\Delta V=V_{k+1}-V_{k}<0$, which is equivalent to

$$
\Delta V \leqslant \eta \varepsilon^{T}(k+1) N^{T} P N \varepsilon(k+1)+\varepsilon^{T}(k) \mathbb{M}_{11} \varepsilon(k)+\Delta g_{k}^{T} \mathbb{M}_{22} \Delta g_{k}+2 \varepsilon^{T}(k) \mathbb{S} \Delta g_{k}+2 \varepsilon^{T}(k+1) N^{T} \mathbb{M}_{23} \Delta g_{k+1}+\Delta g_{k+1}^{T} \mathbb{M}_{33} \Delta g_{k}
$$

Using the dynamics of the estimation error (32) and based on the Lyapunov stability theory, the convergence of the estimation error is guaranteed, as soon as $\Delta V<0$, which holds if

$$
\zeta^{T} \mathcal{M} \zeta<0
$$


where

$$
\zeta^{T}(k)=\left[\begin{array}{lll}
\varepsilon^{T}(k) & \Delta g_{K}^{T} & \Delta g_{K+1}^{T}
\end{array}\right]
$$

and

$$
\mathcal{M}=\left[\begin{array}{ccc}
\mathbb{M}_{11}+\eta \mathcal{M}_{12} P^{-1} \mathcal{M}_{12}^{T} & \eta \mathcal{M}_{12} P^{-1} \widetilde{\mathcal{M}}_{12}^{T}+\mathbb{S} & \mathcal{M}_{12} P^{-1} \mathbb{M}_{23} \\
\star & \eta \widetilde{\mathcal{M}}_{12} P^{-1} \widetilde{\mathcal{M}}_{12}^{T}+\mathbb{M}_{22} & \widetilde{\mathcal{M}}_{12} P^{-1} \mathbb{M}_{23} \\
\star & \star & \mathbb{M}_{33}
\end{array}\right]
$$

with

$$
\left\{\begin{array}{l}
\mathcal{M}_{12}=\left(A_{z}-K C_{z}\right)^{T} N^{T} P \\
\widetilde{\mathcal{M}}_{12}=(L-K C)^{T} N^{T} P
\end{array}\right.
$$

The BMI problem of (38)-(41) is not convex and the existing LMI computational procedures can not be applied. To linearize BMI problem (38)-(41), we proceed in three steps.

The first step concerns the blocks $(1,2),(1,3)$ and $(2,3)$ of matrix $\mathcal{M}$ in relation (40). Since we have $\mathcal{M}_{12}, \widetilde{\mathcal{M}}_{12}$ and $\mathbb{M}_{23}$ acting separately in (40), and since $\mathcal{M}_{12}$ and $\widetilde{\mathcal{M}}_{12}$ are functions of $K$ and $P$, while $\mathbb{M}_{23}$ depends on $S$, it is necessary to rewrite these blocks $(1,2),(1,3)$ and $(2,3)$ under a form so that the matrices $\mathcal{M}_{12}, \widetilde{\mathcal{M}}_{12}$ and $\mathbb{M}_{23}$ appear linearly or quadratically in matrix $\mathcal{M}$. Then the matrix $\mathcal{M}$ is rewritten as follows

$$
\mathcal{M}=\overline{\mathcal{M}}+\Psi \Phi^{T}+\Phi \Psi^{T}
$$

where

$$
\overline{\mathcal{M}}=\left[\begin{array}{ccc}
\mathbb{M}_{11}+\eta \mathcal{M}_{12} P^{-1} \mathcal{M}_{12}^{T} & \mathbb{S} & 0 \\
\star & \eta \widetilde{\mathcal{M}}_{12} P^{-1} \widetilde{\mathcal{M}}_{12}^{T}+\mathbb{M}_{22} & 0 \\
\star & \star & \mathbb{M}_{33}
\end{array}\right]
$$

and

$$
\Psi=\left[\begin{array}{ccc}
\eta \mathcal{M}_{12} & \mathcal{M}_{12} & 0 \\
0 & 0 & \widetilde{\mathcal{M}}_{12} \\
0 & 0 & 0
\end{array}\right], \Phi=\left[\begin{array}{ccc}
0 & 0 & 0 \\
\widetilde{\mathcal{M}}_{12} P^{-1} & 0 & 0 \\
0 & \mathbb{M}_{23}^{T} P^{-1} & \mathbb{M}_{23}^{T} P^{-1}
\end{array}\right]
$$

In the second step, we use inequality (21) to obtain an upper bound of the term $\Psi \Phi^{T}+\Phi \Psi^{T}$ in (42) and the following inequality holds for any positive scalar $\epsilon$

$$
\Psi \Phi^{T}+\Phi \Psi^{T} \leqslant \epsilon\left[\begin{array}{ccc}
0 & 0 & 0 \\
\widetilde{\mathcal{M}}_{12} & 0 & 0 \\
0 & \mathbb{M}_{23}^{T} & \mathbb{M}_{23}^{T}
\end{array}\right] P^{-2}\left[\begin{array}{ccc}
0 & \widetilde{\mathcal{M}}_{12}^{T} & 0 \\
0 & 0 & \mathbb{M}_{23} \\
0 & 0 & \mathbb{M}_{23}
\end{array}\right]+\frac{1}{\epsilon}\left[\begin{array}{ccc}
\eta \mathcal{M}_{12} & \mathcal{M}_{12} & 0 \\
0 & 0 & \widetilde{\mathcal{M}}_{12} \\
0 & 0 & 0
\end{array}\right]\left[\begin{array}{ccc}
\eta \mathcal{M}_{12}^{T} & 0 & 0 \\
\mathcal{M}_{12}^{T} & 0 & 0 \\
0 & \widetilde{\mathcal{M}}_{12}^{T} & 0
\end{array}\right] \text {. }
$$

From the fact that $P^{-2}<\alpha^{-2} I$ (since $P>\alpha I$ ), it follows that

$$
\mathcal{M} \leqslant \widetilde{\mathcal{M}}+\left[\begin{array}{ccc}
\frac{1+\eta^{2}}{\epsilon} \mathcal{M}_{12} \mathcal{M}_{12}^{T} & 0 & 0 \\
0 & \frac{1+\epsilon^{2} \alpha^{-2}}{\epsilon} \widetilde{\mathcal{M}}_{12} \widetilde{\mathcal{M}}_{12}^{T} & 0 \\
0 & 0 & 2 \epsilon \alpha^{-2} \mathbb{M}_{23}^{T} \mathbb{M}_{23}
\end{array}\right]=\mathbf{M}
$$

This implies that if the linear matrix inequality

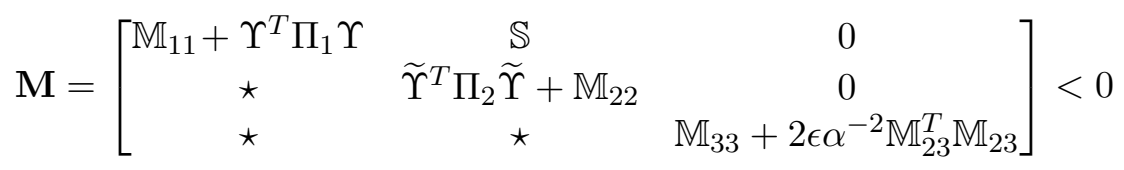

is verified then $\Delta V<0$, where

$$
\left\{\begin{array}{l}
\Upsilon^{T}=\left[\begin{array}{ll}
\mathcal{M}_{12} & \mathcal{M}_{12}
\end{array}\right] \\
\widetilde{\Upsilon}^{T}=\left[\begin{array}{ll}
\widetilde{\mathcal{M}}_{12} & \widetilde{\mathcal{M}}_{12}
\end{array}\right] \\
\Pi_{1}=\left[\begin{array}{cc}
\eta P^{-1} & 0 \\
0 & \frac{\eta^{2}+1}{\epsilon} I_{n}
\end{array}\right] \\
\Pi_{2}=\left[\begin{array}{cc}
\eta P^{-1} & 0 \\
0 & \frac{1+\epsilon^{2} \alpha^{-2}}{\epsilon} I_{n}
\end{array}\right]
\end{array}\right.
$$


Now using the Schur lemma [22], the inequality (46) is equivalent to the LMI

$$
\left[\begin{array}{cccccc}
\mathbb{M}_{11} & \mathbb{S} & 0 & \Upsilon^{T} & 0 & 0 \\
\star & \mathbb{M}_{22} & 0 & 0 & \widetilde{\Upsilon}^{T} & 0 \\
\star & \star & \mathbb{M}_{33} & 0 & 0 & \mathbb{M}_{23}^{T} \\
\star & \star & \star & -\Pi_{1}^{-1} & 0 & 0 \\
\star & \star & \star & \star & -\Pi_{2}^{-1} & 0 \\
\star & \star & \star & \star & \star & \frac{-1}{2} \epsilon^{-1} \alpha^{2} I_{n}
\end{array}\right]<0 .
$$

By using the notation $X=K^{T} N^{T} P$, the LMIs in (34) and (48) are equivalent, which completes the proof.

\section{Numerical Example}

In this section, we present two illustrative examples showing the application of both theorems given in sections 2 and 3 respectively.

\subsection{First example}

Consider the following discrete-time model of a moving object in the cartesian coordinates [20]:

$$
\left\{\begin{array}{l}
\dot{x}(t)=A_{c} x(t)+f_{c}(x(t), y(t)) \\
y(t)=C_{c} x(t)
\end{array}\right.
$$

where

$$
A_{c}=\left[\begin{array}{cc}
1 & -1 \\
1 & 1
\end{array}\right], C_{c}=\left[\begin{array}{ll}
0 & 1
\end{array}\right]
$$

The nonlinear function $f_{c}(x, y)$ is given by

$$
f_{c}(x, y)=\left[\begin{array}{l}
-x_{1}\left(x_{1}^{2}+x_{2}^{2}\right) \\
-x_{2}\left(x_{1}^{2}+x_{2}^{2}\right)
\end{array}\right]
$$

By applying the Euler method on system (49), a discrete time system is derived as follows

$$
\left\{\begin{aligned}
x(k+1) & =A x(k)+f(x(k), y(k)) \\
y(k) & =C x(k)
\end{aligned}\right.
$$

where $A=I_{2}+T_{e} A_{c}, f(x(k), y(k))=T_{e} f_{c}(x(t), y(t))$ and $C_{c}=C$.

First, we verify the one-sided Lipschitz property of the function $f$

$$
\langle f(x, y)-f(\hat{x}, y), x-\hat{x}\rangle=T_{e}\left(x_{1} \hat{x}_{1}+x_{2} \hat{x}_{2}\right)\left(\left(x_{1}^{2}+x_{2}^{2}\right)+\left(\hat{x}_{1}^{2}+\hat{x}_{2}^{2}\right)\right)-T_{e}\left(x_{1}^{2}+x_{2}^{2}\right)^{2}-T_{e}\left(\hat{x}_{1}^{2}+\hat{x}_{2}^{2}\right)^{2} .
$$

On the other hand

$$
\begin{aligned}
x_{1} \hat{x}_{1}+x_{2} \hat{x}_{2} & =\frac{1}{2}\left(x_{1}^{2}+x_{2}^{2}\right)+\frac{1}{2}\left(\hat{x}_{1}^{2}+\hat{x}_{2}^{2}\right)-\frac{1}{2}\left(x_{1}-\hat{x}_{1}\right)^{2}-\frac{1}{2}\left(x_{2}-\hat{x}_{2}\right)^{2} \\
& =\frac{1}{2}\left(\|x\|^{2}+\|\hat{x}\|^{2}-\|x-\hat{x}\|^{2}\right) .
\end{aligned}
$$

Inserting (52) into (51) leads to the following equation

$$
\begin{aligned}
\langle f(x, y)-f(\hat{x}, y), x-\hat{x}\rangle= & \frac{T_{e}}{2}\left(\|x\|^{2}+\|\hat{x}\|^{2}-\|x-\hat{x}\|^{2}\right)\left(\|x\|^{2}+\|\hat{x}\|^{2}\right)-T_{e}\|x\|^{4}-T_{e}\|\hat{x}\|^{4} \\
& =\frac{-T_{e}}{2}\|x-\hat{x}\|^{2}\left(\|x\|^{2}+\|\hat{x}\|^{2}\right)-\frac{T_{e}}{2}\left(\|x\|^{2}-\|\hat{x}\|^{2}\right)^{2} \\
& \leqslant 0
\end{aligned}
$$


Thus, $f$ satisfies globally the one-sided Lipschitz condition (2) with an one-sided Lipschitz constant $\rho=0$. Now, let us verify the Lipschitz continuity property. According to the mean-value theorem and since $f$ is of class $\mathcal{C}^{1}$, we have

$$
f(x, y)-f(\hat{x}, y)=J_{f}(x, \hat{x}, y) e
$$

where $J_{f}$ is the Jacobian matrix of $f$ defined by

$$
J_{f}=T_{e}\left[\begin{array}{cc}
-3 x_{1}^{2}-x_{2}^{2} & -2 x_{1} x_{2} \\
-2 x_{1} x_{2} & -3 x_{2}^{2}-x_{1}^{2}
\end{array}\right]
$$

By the fact that $J_{f}$ is a symmetric matrix, then its induced 2-norm equals its spectral radius

$$
\left\|J_{f}\right\| \triangleq \max _{1 \leqslant i \leqslant 2}\left|\lambda_{i}\left(J_{f}\right)\right|=3 T_{e}\left(x_{1}^{2}+x_{2}^{2}\right) \leqslant 3 T_{e} r^{2} .
$$

Let $\mathcal{D}=\left\{x \in \mathbb{R}^{2}:\|x\| \leqslant r\right\}$ denote the domains of functioning of $x$. A simple reasoning shows that

$$
\|f(x, y)-f(\hat{x}, y)\| \leqslant 3 T_{e} r^{2}\|e\|, \quad \forall x \in \mathcal{D} .
$$

This means that the system is locally Lipschitz and the Lipschitz constant is $3 T_{e} r^{2}$ on any set $\mathcal{D}$.

Finally, we verify the quadratic inner-boundedness property of the system. First, we develop the left hand-side of the inequality (3)

$$
\begin{aligned}
\|f(x, y)-f(\hat{x}, y)\|^{2} & =T_{e}^{2}\left(-x_{1}\left(x_{1}^{2}+x_{2}^{2}\right)+\hat{x}_{1}\left(\hat{x}_{1}^{2}+\hat{x}_{2}^{2}\right)\right)^{2}+T_{e}^{2}\left(-x_{2}\left(x_{1}^{2}+x_{2}^{2}\right)+\hat{x}_{2}\left(\hat{x}_{1}^{2}+\hat{x}_{2}^{2}\right)\right)^{2} \\
& =T_{e}^{2}\left(x_{1}^{2}+x_{2}^{2}\right)^{3}+T_{e}^{2}\left(\hat{x}_{1}^{2}+\hat{x}_{2}^{2}\right)^{3}-2 T_{e}^{2}\left(x_{1}^{2}+x_{2}^{2}\right)\left(\hat{x}_{1}^{2}+\hat{x}_{2}^{2}\right)\left(x_{1} \hat{x}_{1}+x_{2} \hat{x}_{2}\right) \\
& =T_{e}^{2}\|x\|^{6}+T_{e}^{2}\|\hat{x}\|^{6}-T_{e}^{2}\|x\|^{2}\|\hat{x}\|^{2}\left(\|x\|^{2}+\|\hat{x}\|^{2}-\|x-\hat{x}\|^{2}\right) \\
& =T_{e}^{2}\left(\|x\|^{2}-\|\hat{x}\|^{2}\right)\left(\|x\|^{4}-\|\hat{x}\|^{4}\right)+T_{e}^{2}\|x\|^{2}\|\hat{x}\|^{2}\|x-\hat{x}\|^{2} \\
& =T_{e}^{2}\left(\|x\|^{2}-\|\hat{x}\|^{2}\right)^{2}\left(\|x\|^{2}+\|\hat{x}\|^{2}\right)+T_{e}^{2}\|x\|^{2}\|\hat{x}\|^{2}\|x-\hat{x}\|^{2} .
\end{aligned}
$$

Second, using the equation (53), the right hand-side of the of the inequality (3) becomes

$\gamma\langle f(x, y)-f(\hat{x}, y), x-\hat{x}\rangle+\beta\|x-\hat{x}\|^{2}=-\frac{\gamma}{2} T_{e}\|x-\hat{x}\|^{2}\left(\|x\|^{2}+\|\hat{x}\|^{2}\right)-\frac{\gamma}{2} T_{e}\left(\|x\|^{2}-\|\hat{x}\|^{2}\right)^{2}+\beta\|x-\hat{x}\|^{2}$.

The expressions (58) and (59) fulfill the condition (3) if the following inequalities are verified

$$
\left\{\begin{aligned}
\|x\|^{2}+\|\hat{x}\|^{2} & \leqslant \frac{-\gamma}{2 T_{e}} \\
\|x\|^{2}\|\hat{x}\|^{2} & \leqslant \frac{\beta}{T_{e}^{2}}-\frac{\gamma}{2 T_{e}}\left(\|x\|^{2}+\|\hat{x}\|^{2}\right) \leqslant \frac{\beta}{T_{e}^{2}}+\frac{\gamma^{2}}{4 T_{e}^{2}}
\end{aligned}\right.
$$

for all $x \in \mathcal{D}$ and $\hat{x} \in \mathcal{D}$. So we conclude that the quadratic inner-bounded condition (3) holds if

$$
\left\{\begin{aligned}
2 r^{2} & \leqslant \frac{-\gamma}{2 T_{e}} \\
r^{4} & \leqslant \frac{\beta}{T_{e}^{2}}+\frac{\gamma^{2}}{4 T_{e}^{2}}
\end{aligned}\right.
$$

consequently if

$$
r=\min \left(\left(\frac{-\gamma}{4 T_{e}}\right)^{1 / 2},\left(\frac{\beta}{T_{e}^{2}}+\frac{\gamma^{2}}{4 T_{e}^{2}}\right)^{1 / 4}\right), \quad \gamma \leqslant 0, \beta \geqslant \frac{-\gamma^{2}}{4} .
$$

Then the quadratically inner-bounded property of $f(x, y)$ is verified in $\mathcal{D}$. As the system is globally one-sided Lipschitz, i.e., $D=\mathbb{R}^{2}, D \cap \mathcal{D}=\mathcal{D}$. Note that the region $\mathcal{D}$ can be made arbitrarily large by 
choosing appropriate values for $\gamma$ and $\beta$. Notice that to maximize $r$ in relation (62) for a given $\gamma$, the parameter $\beta$ should satisfy

$$
\left(\frac{-\gamma}{4 T_{e}}\right)^{1 / 2} \leqslant\left(\frac{\beta}{T_{e}^{2}}+\frac{\gamma^{2}}{4 T_{e}^{2}}\right)^{1 / 4}
$$

which is equivalent to

$$
\beta \geqslant \frac{-3 \gamma^{2}}{16}
$$

If we chose $\beta=1$ and $\gamma=-256$, we get $r=8$. The LMI-based observer gain matrix is

$$
K=\left[\begin{array}{c}
-0.1563 \\
1.0945
\end{array}\right]
$$

However, we propose to use the reduced-order version. With $L=\left[\begin{array}{ll}1 & 0\end{array}\right]$, we have $N=\left[\begin{array}{ll}1 & 0\end{array}\right]^{T}$ and $M=\left[\begin{array}{ll}0 & 1\end{array}\right]^{T}$. The LMI-based observer gain matrix is

$$
K=0.1993 .
$$

Simulations were processed with the sample time $T_{e}=0.1$ [sec]. As shown in figures 1 to 3 , the state is very well estimated.

\subsection{Second example}

The goal of this section is to apply the theoretical results introduced in the previous sections to the example used in [6] that is the one-link flexible joint robot [24]

$$
\left\{\begin{array}{l}
\dot{\theta}_{m}=\omega_{m} \\
\dot{\omega}_{m}=\frac{\kappa}{J_{m}}\left(\theta_{l}-\theta_{m}\right)-\frac{b}{J_{m}} \omega_{m}+\frac{K_{T}}{J_{m}} u \\
\dot{\theta}_{l}=\omega_{1} \\
\dot{\omega}_{l}=\frac{\kappa}{J_{l}}\left(\theta_{l}-\theta_{m}\right)-\frac{m g h}{J_{l}} \sin \left(\theta_{l}\right)
\end{array}\right.
$$

where $\theta_{m}$ and $\theta_{l}$ are the angles of rotations of the motor and link, respectively. $\omega_{m}$ and $\omega_{l}$ are their angular velocities. $J_{m}$ and $J_{l}$ are the inertia of the motor and link respectively. $K_{T}, \kappa, m, g$ and $h$ are positive constants.

The behavior of the link flexible joint robot can be described by nonlinear state-space equations (1) where $x=\left[\begin{array}{llll}\theta_{m} & \omega_{m} & \theta_{l} & \omega_{l}\end{array}\right]^{T}$. The nonlinear function $f(x)$ is given by $f(x)=3.205 \sin \left(x_{3}\right)$. We consider that the measurements of the system are the angle of rotation of the motor $\theta_{m}$ and the angular velocity $\omega_{m}$. The system matrices can be computed with the numerical values of the link flexible joint robot parameters available in $[6]$

$$
\begin{aligned}
& \dot{x}=\underbrace{\left[\begin{array}{cccc}
-10 & 1 & 0 & 0 \\
-48.6 & -1.26 & 48.6 & 0 \\
0 & 0 & -22 & 1 \\
1.95 & 0 & -19.5 & -6
\end{array}\right]}_{A_{c}} x+\underbrace{\left[\begin{array}{l}
0 \\
1 \\
0 \\
0
\end{array}\right]}_{B_{c}} u+\underbrace{\left[\begin{array}{c}
0 \\
0 \\
0 \\
3.205 \sin \left(x_{3}\right)
\end{array}\right]}_{f_{c}(x(t), y(t))} \\
& y=\underbrace{\left[\begin{array}{llll}
1 & 0 & 0 & 0 \\
0 & 1 & 0 & 0
\end{array}\right]}_{C_{c}} x .
\end{aligned}
$$

The Euler discretization of this dynamic system gives

$$
\left\{\begin{aligned}
x(k+1) & =A x(k)+B u(k)+f(x(k), y(k)) \\
y(k) & =C x(k)
\end{aligned}\right.
$$


where $A=I_{4}+T_{e} A_{c}, B=T_{e} B_{c}, f(x(k), y(k))=T_{e} f_{c}(x(t), y(t))$ and $C=C_{c}$. Using the mean value theorem, It is easy to verify that $f(x(k), y(k))$ satisfies condition $(2)$ with one-sided Lipschitz constant $\rho=3.205 T_{e}$. In addition, $f(x(k), y(k))$ is Lipschitz function, then, is also quadratically inner-bounded with $\beta=3.205 T_{e}$ and $\gamma=0$.

The LMI-based observer gain matrix is

$$
K=\left[\begin{array}{cc}
0.5790 & 0.01 \\
-0.4860 & 0.7421 \\
0 & 0.1128 \\
0.0195 & -0.1797
\end{array}\right]
$$

However, we propose to use the reduced-order version. With $L=\left[\begin{array}{llll}0 & 0 & 1 & 0 \\ 0 & 0 & 0 & 1\end{array}\right]$, we have $N=\left[\begin{array}{llll}0 & 0 & 1 & 0 \\ 0 & 0 & 0 & 1\end{array}\right]^{T}$ and $M=\left[\begin{array}{cccc}1 & 0 & 0 & 0 \\ 0 & 1 & 0 & 0\end{array}\right]^{T}$. The LMI-based observer gain matrix is

$$
K=\left[\begin{array}{cc}
0 & 0.7610 \\
0 & -0.4323
\end{array}\right]
$$

Simulations were processed with the sample time $T_{e}=0.1$ [sec]. As shown in figures 4 to 9 , the state is very well estimated.

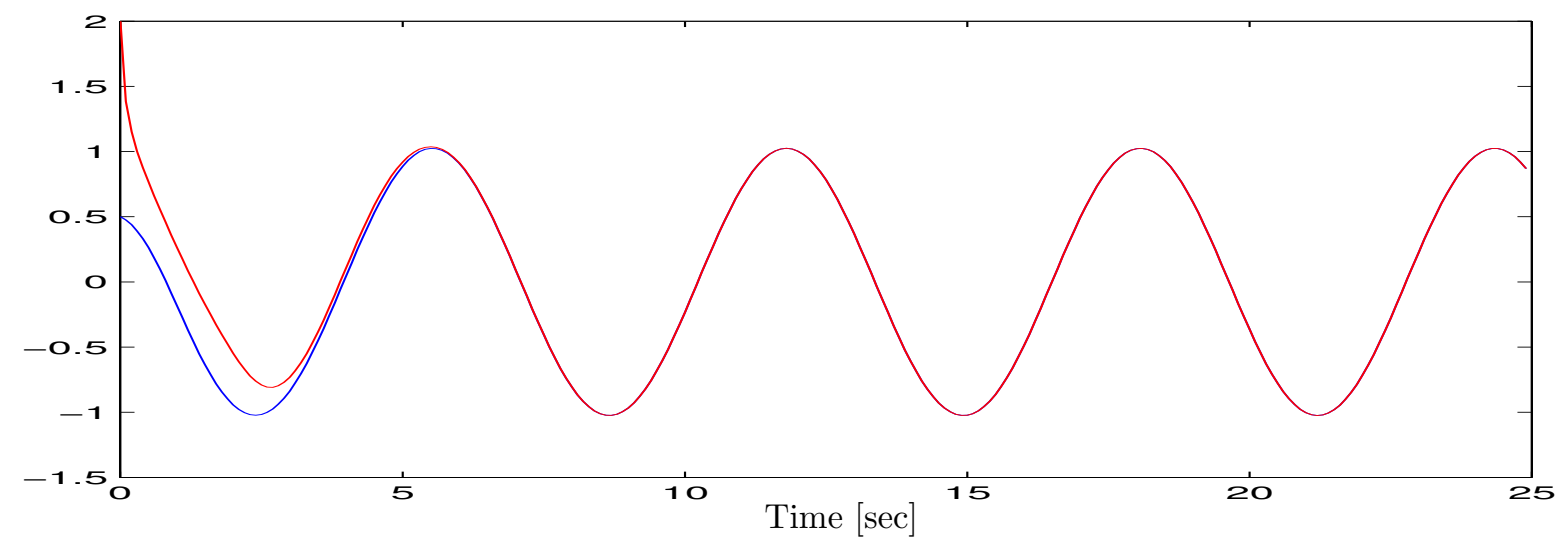

Figure 1: Example 1: response of $x_{1}(k)$ (blue line) and its estimate with the full-order observer (red line).

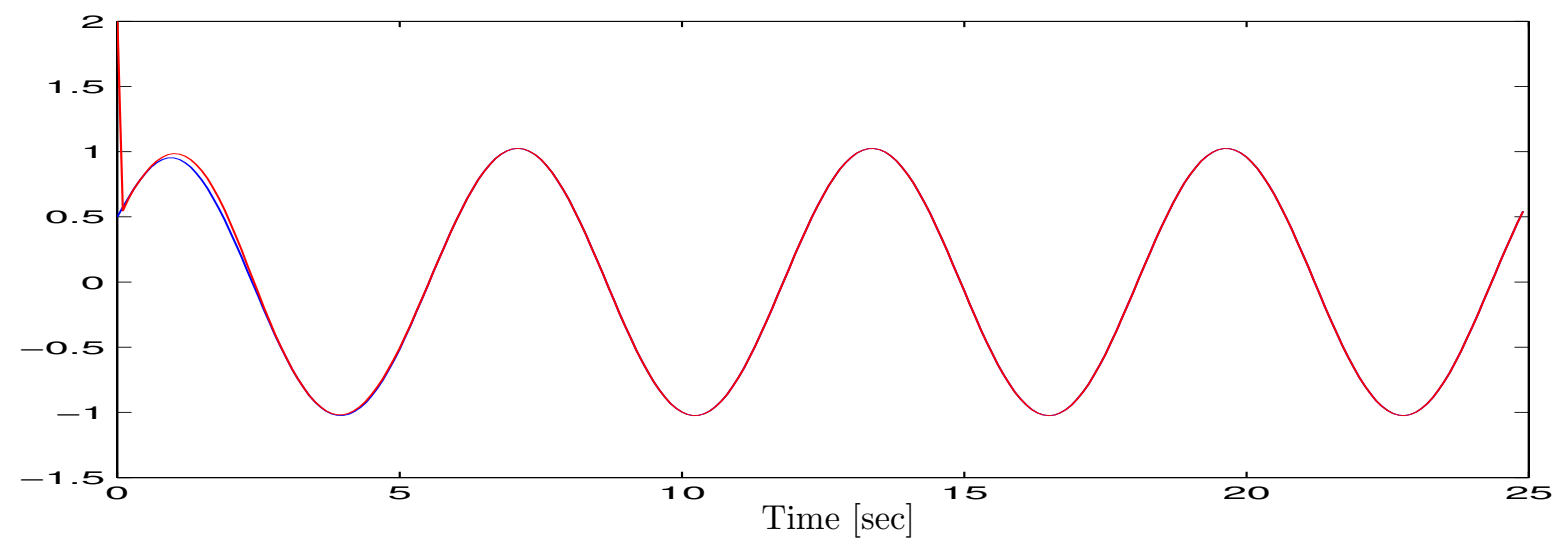

Figure 2: Example 1: response of $x_{2}(k)$ (blue line) and its estimate with the full-order observer.

\section{Conclusion}

In this note, we proposed a simple and useful observer design method for a class of nonlinear discrete-time systems that satisfies the one-sided Lipschitz condition. One of the main challenges we tackled, using 


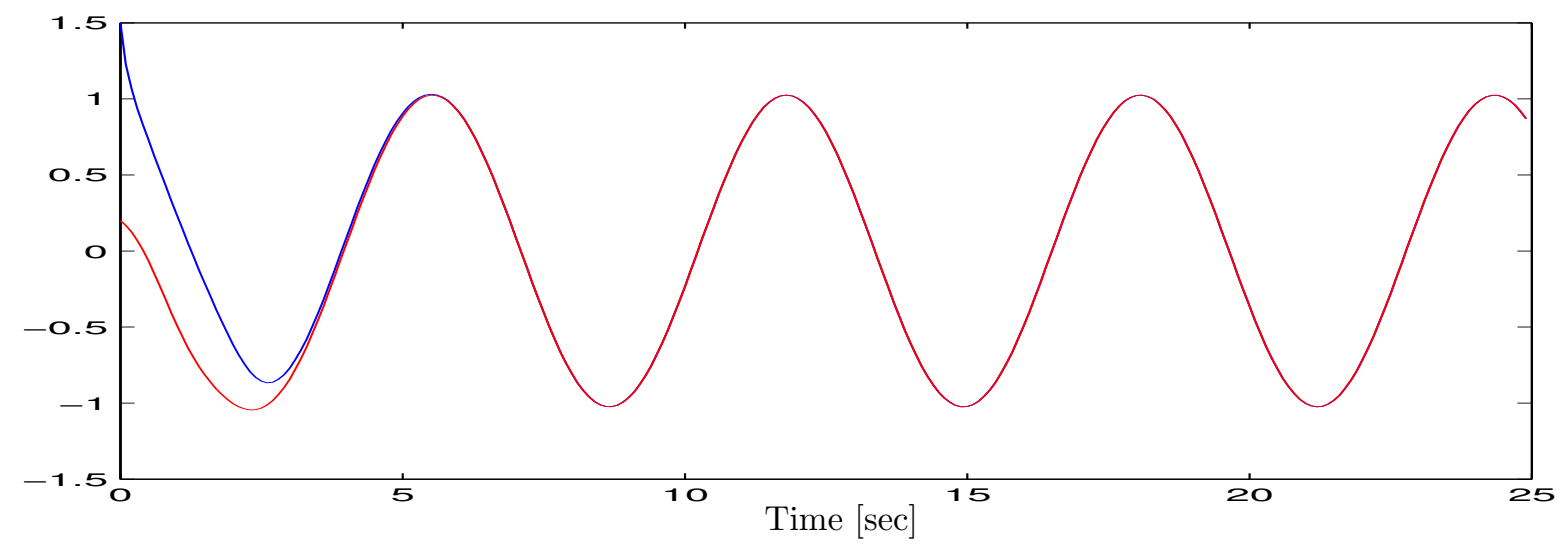

Figure 3: Example 1 : response of $x_{1}(k)$ (blue line) and its estimate with the reduced-order observer (red line).

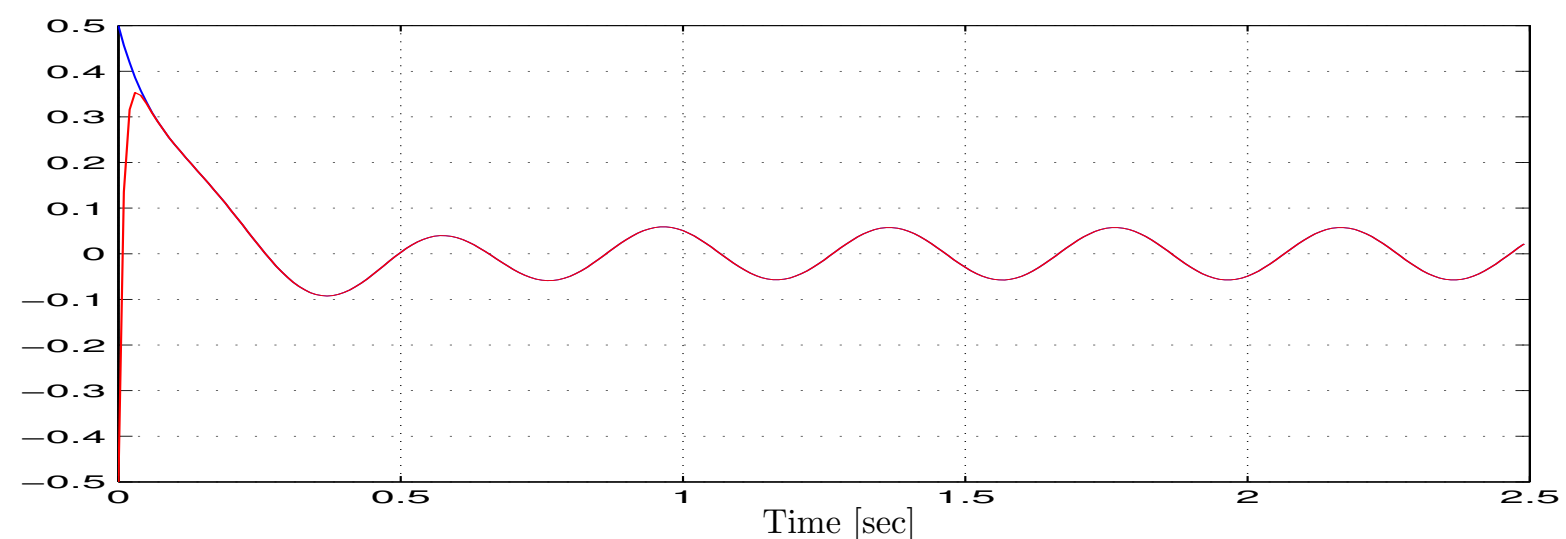

Figure 4: Example 2: response of $x_{1}(k)$ (blue line) and its estimate with the full-order observer (red line).

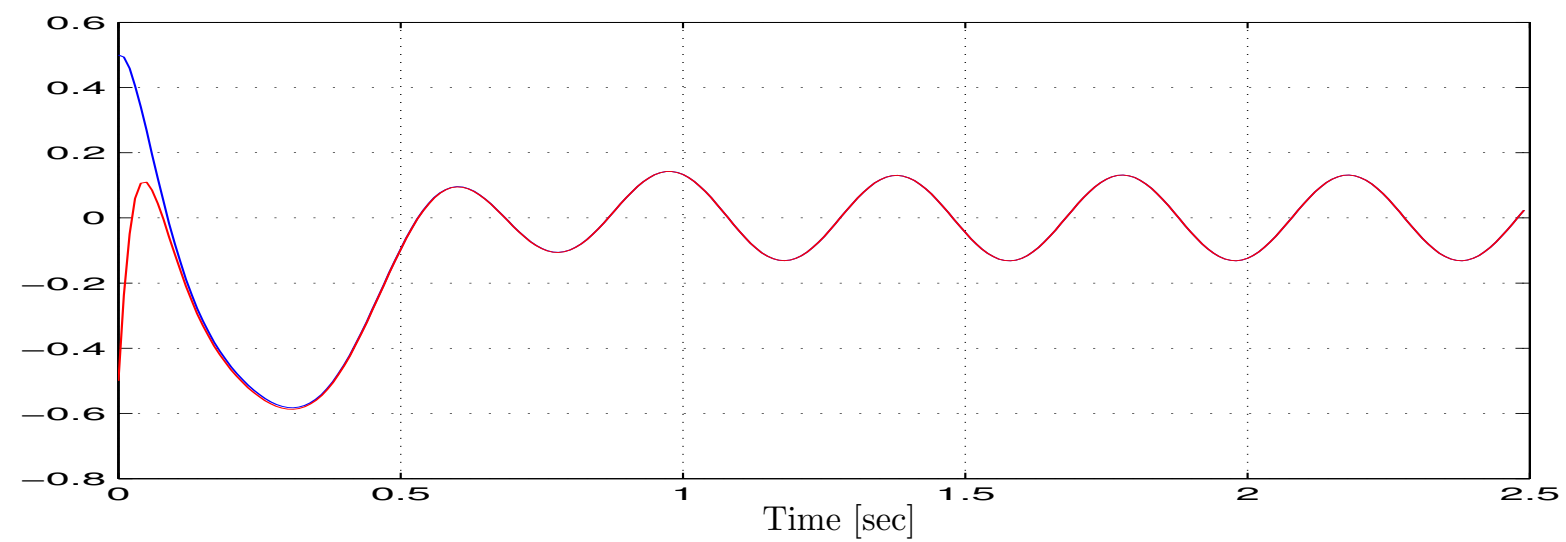

Figure 5: Example 2 : response of $x_{2}(k)$ (blue line) and its estimate with the full-order observer (red line).

several artifacts, is to provide LMI conditions for the asymptotic convergence. On the other hand, we considered a more general Lyapunov function in which we introduced nonlinearities of the system. This function has the advantage of reducing the complexity of the obtained conditions. Furthermore, both full-order and reduced-order observer designs were considered. Illustrative examples were given in order to show the high performances of the proposed methods.

\section{References}

[1] A. Krener and A. Isidori, "Linearization by output injection and nonlinear observers," Syst. E Contr. Letters, vol. 3, pp. 47-52, 1983. 


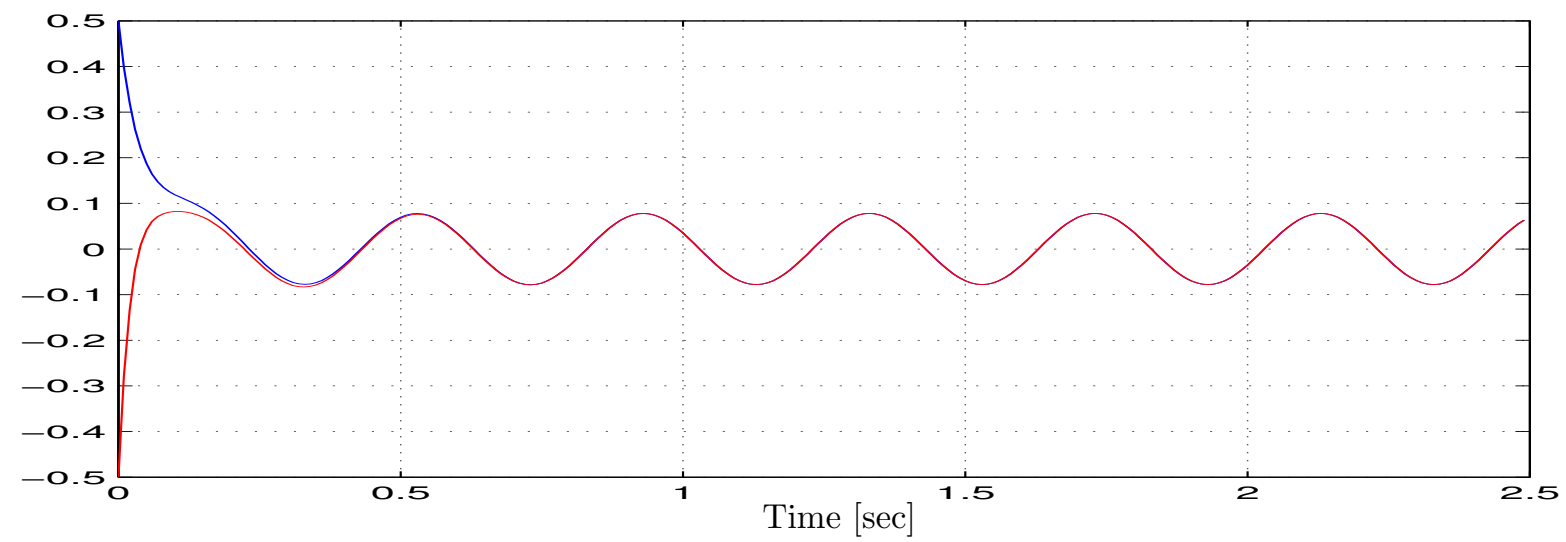

Figure 6: Example 2: response of $x_{3}(k)$ (blue line) and its estimate with the full-order observer (red line).

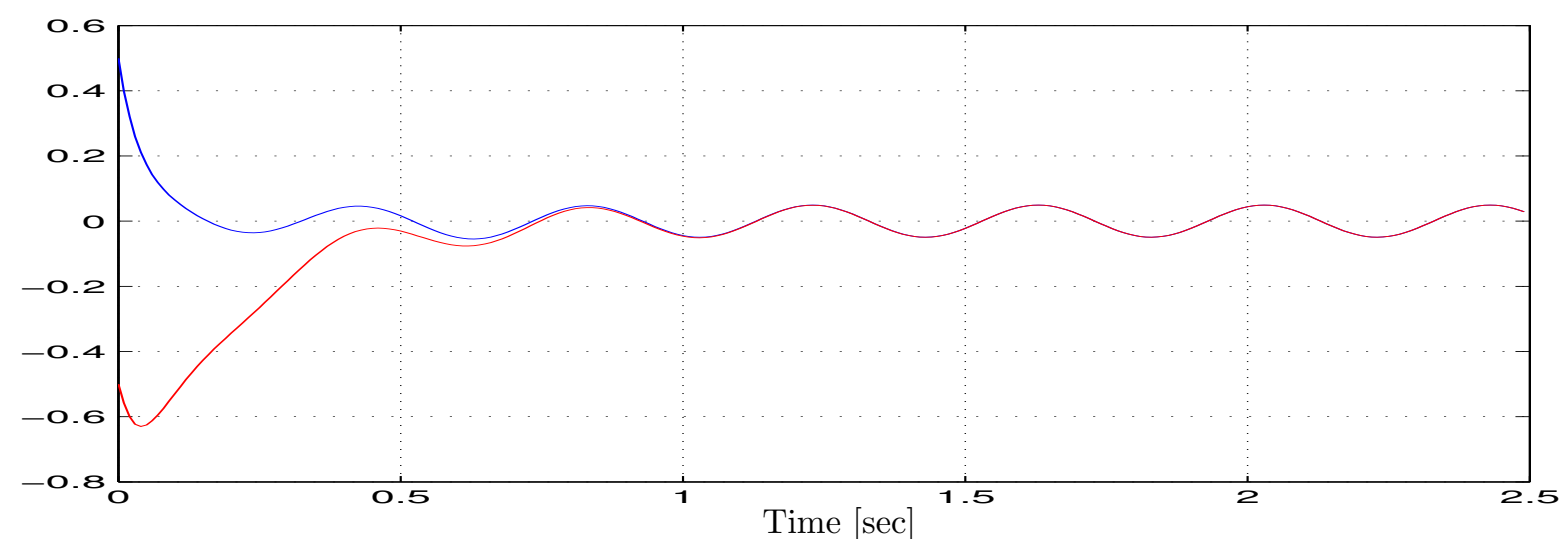

Figure 7: Example 2: response of $x_{4}(k)$ (blue line) and its estimate with the full-order observer (red line).

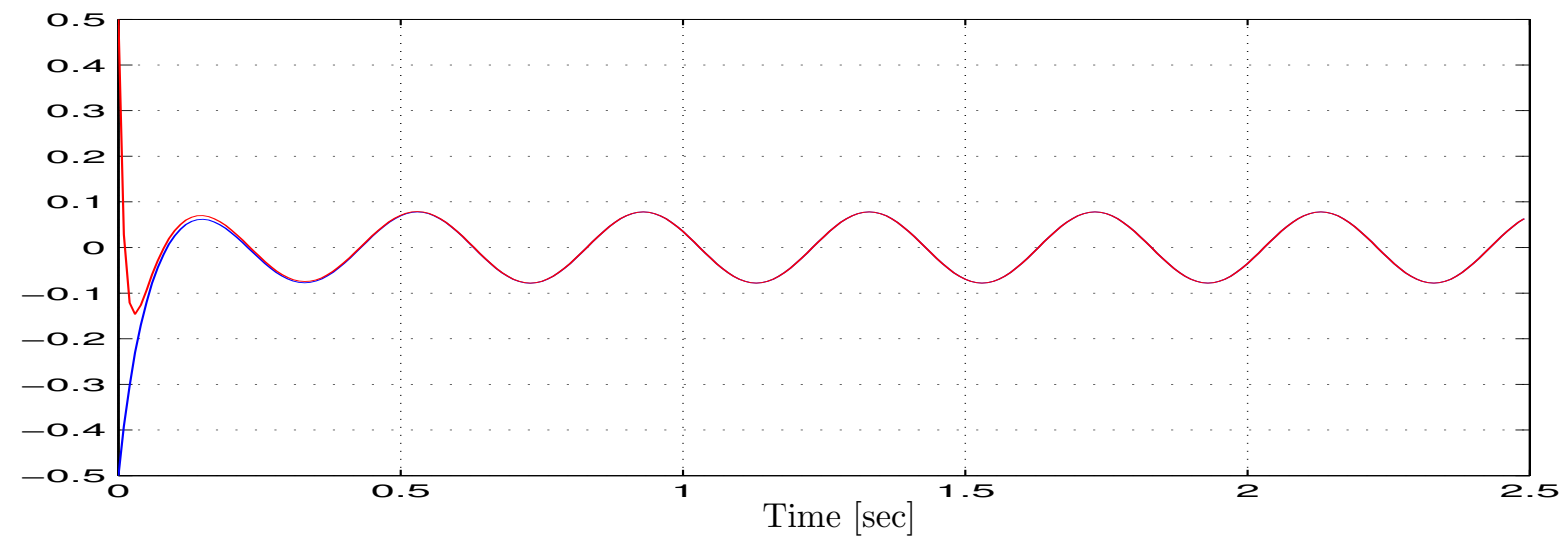

Figure 8: Example 2: response of $x_{3}(k)$ (blue line) and its estimate with the reduced-order observer (red line).

[2] X. Xia and W. Gao, "Nonlinear observer design by observer error linearization," SIAM J. Contr. Opt., vol. 27, pp. 199-216, 1989.

[3] M. Hou and A. Pugh, "Observer with linear error dynamics for nonlinear multi-output systems," Syst. $\&$ Contr. Letters, vol. 37, pp. 1-9, 1999.

[4] M. Boutayeb and M. Darouach, "A reduced-order observer for non-linear discrete-time systems," Syst. $\&$ Contr. Letters, vol. 39, pp. 141-151, 2000.

[5] S. Ibrir, "Circle-criterion approach to discrete-time nonlinear observer design," Automatica, vol. 43, pp. 1432-1441, 2007. 


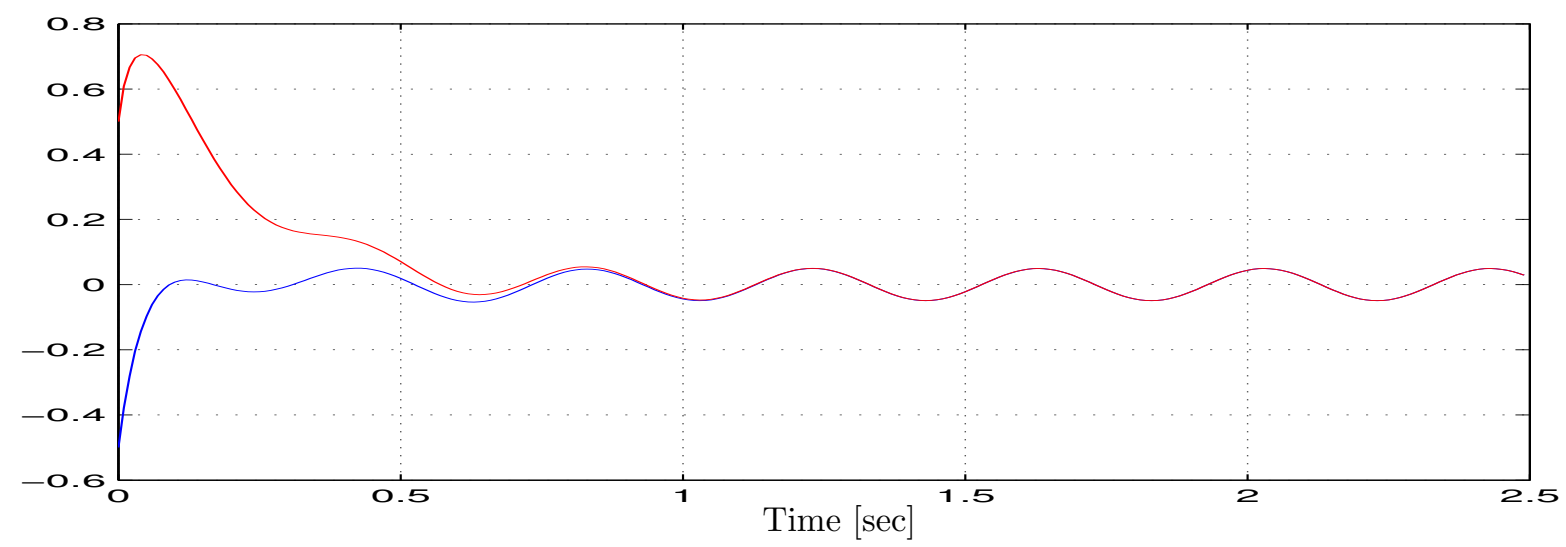

Figure 9: Example 2: response of $x_{4}(k)$ (blue line) and its estimate with the reduced-order observer (red line).

[6] Q. Ha and H. Trinh, "State and input simultaneous estimation for a class of nonlinear systems," Automatica, vol. 40, pp. 1779-1785, 2004.

[7] D. Dawson, Z. Qu, and J. Carroll, "On the state observation and output feedback problems for nonlinear uncertain dynamic systems," Syst. \& Contr. Letters, vol. 18, pp. 217-222, 1992.

[8] M. Boutayeb, "Synchronization and input recovery in digital nonlinear systems," IEEE Trans. Circ. Syst. II : Express Briefs, vol. 49, pp. 393-399, 2004.

[9] W. Lee and K. Nam, "Observer design for autonomous discrete-time nonlinear systems," Syst. \& Contr. Letters, vol. 17, pp. 49-58, 1991.

[10] W. Lin and C. Byrnes, "Remarks on linearization of discrete-time autonomous systems and nonlinear observer design," Syst. \& Contr. Letters, vol. 25, pp. 31-40, 1995.

[11] F. Thau, "Observing the state of non-linear dynamic systems," Int. J. Contr., vol. 17, pp. 471-479, 1973.

[12] R. Rajamani, "Observer for Lipschitz nonlinear systems," IEEE Trans. Aut. Contr., vol. 43, pp. 397401, 1998.

[13] G. Phanomchoeng and R. Rajamani, "The bounded Jacobian approach to nonlinear observer design," in Proc. IEEE American Contr. Conf., (Baltimore, USA), 2010.

[14] A. Zemouche, M. Boutayeb, and G. Bara, "Observers for a class of Lipschitz systems with extension to $\mathcal{H}_{\infty}$ performance analysis," Syst. $\mathcal{G}$ Contr. Letters, vol. 57, pp. 18-27, 2008.

[15] G. Hu, "Observers for one-sided Lipschitz non-linear systems," IMA J. Mathematical Control $\&$ Information, vol. 23, pp. 395-401, 2006.

[16] G. Hu, "A note on observer for one-sided Lipschitz non-linear systems," IMA J. Mathematical Control \& Information, vol. 25, pp. 297-303, 2008.

[17] Z. Tai and X. Wang, "H⿻ $\boldsymbol{H}_{\infty}$ observers for one-sided lipschitz nonlinear systems," in Proc. IEEE World Congress on Intelligent Control and Automation, (Chongqing, P.R. China), 2008.

[18] M. Xu, G. Hu, and Y. Zhao, "Reduced-order observer design for one-sided Lipschitz non-linear systems," IMA J. Mathematical Control \& Information, vol. 26, pp. 299-307, 2009.

[19] Y. Zhao, J. Tao, and N. Shi, "A note on observer design for one-sided Lipschitz nonlinear systems," Syst. E Contr. Letters, vol. 59, pp. 66-71, 2010.

[20] M. Abbaszadeh and H. Marquez, "Nonlinear observer design for one-sided Lipschitz systems," in Proc. IEEE American Contr. Conf., (Baltimore, USA), 2010. 
[21] I. Petersen, "A stabilization algorithm for a class of uncertain linear systems," Syst. E Contr. Letters, vol. 8, pp. 351-357, 1987.

[22] S. Boyd, L. El Ghaoui, E. Féron, and V. Balakrishnan, Linear Matrix Inequalities in Systems and Control Theory. Philadelphia: SIAM, 1994.

[23] H. Trinh and T. Fernando, Functional Observers for Dynamical Systems, vol. 420 of Lecture Notes in Control and Information Sciences. Heidelberg: Springer-Verlag, 2012.

[24] M. Spong, "Modeling and control of elastic joint robots," Trans. of the ASME, J. Dyn. Syst., Meas. \& Cont., vol. 109, pp. 310-319, 1987. 